\title{
Book Reviews Section
}

This is the first issue that will include a Book Reviews section and this will be a permanent addition to the journal. From time to time publishers will ask for a review to be carried out of a new text and a copy will be supplied to the Book Reviews Editor; a request for a reviewer will then be made through this page. Of course, the reviewer will be entitled to keep any book reviewed. Suggestions for book reviews of current useful texts are welcomed and all enquiries should be made to the address below. The review format should follow that which is published by Jemima Koles in this issue.

The Journal is currently seeking reviewers for the following texts:

1. Turning Points: An Extraordinary Journey into the Suicidal Mind and Confidentiality for Mental Health Professionals by Diego de Lego. Published by Australian Academic Press

2. The Bravehearts Toolbox - for Practitioners Working with Child Sexual Assault by McKillop, Ronken, \& Vidler. Published by Australian Academic Press.

3. Educational Psychology: First Australian Edition by O’ Donnell et al. Published by Wiley.

If you are interested in reviewing one of the above then please send your details by email.

Gerald Wurf, Charles Sturt University, Australia

Book Reviews Editor

gwurf@csu.edu.au

\section{Cognitive Behaviour Therapy: Basics and Beyond (2nd ed.)}

Edited by Judith S. Beck

Guilford Press, 2011, 386 pp., \$50.00 (hardcover), ISBN: 9781609185046.

doi: 10.1017/edp.2012.4

This text provides readers with fresh insights into different cognitive behavioural techniques used to conceptualise and treat clients. It addresses the fundamentals of Cognitive Behaviour Therapy (CBT) while being suitable for a broad audience, from individuals who have never encountered CBT to established practitioners interested in refining their skills. The book has 21 chapters that describe how to conceptualise clients cognitively, plan treatment, employ various cognitive behavioural techniques, assess the effectiveness of their treatment, and specify problems that arise in a therapy session. Each chapter begins with an introduction to the areas that will be covered within the chapter and the learning outcomes. 
Coming from the perspective of a new psychologist, this book provides a comprehensive introduction and evaluation of the current techniques used in cognitive behaviour therapy. It describes the evaluation session and the layout of the subsequent first therapy session in detail and provides ideas for linking the information that was gathered together to form a cognitive conceptualisation. Once the client assessment is explained, you are introduced to the setting of the initial goals for intervention, relating your intervention plan and how to define the expectations for the intervention. Session 2 is also described in detail, and suggestions for future sessions are explained. Additionally, problems associated with having structure in a therapeutic session, such as harming client-therapist rapport and the therapeutic alliance, are discussed in the context of current therapeutic practice. Therapeutic areas of cognitive behaviour therapy, including the identification, evaluation, and ways of responding to automatic thoughts, intermediate beliefs, core beliefs, and additional techniques, are discussed in detail.

With its overview and in-depth update on the therapeutic application of cognitive behaviour therapy, the text will be of use to cognitive behavioural beginners and professionals alike. The techniques covered in this text would be useful in a wide variety of settings, such as with adolescents in a school setting or adults in the general population with severe mood disorders. This book provides a useful update on the current body of knowledge regarding cognitive behaviour therapy and provides an invaluable summary of the techniques currently supported by research. As a result, it is highly recommended for practising psychologists.

Jemima Koles

Provisional Psychologist, Eastern Melbourne Region, Australia

\section{The Social Cure: Identity Health and Wellbeing}

Edited by Jolanda Jetten, Catherine Haslam and S. Alexander Haslam (2012)

Psychology Press, 390 pp., \$53.36 (hardcover), ISBN: 978-1848720213.

doi: 10.1017/edp.2012.5

The term 'theory to practice gap' is often used when describing a disparity between research results and practical observations. At first glance, it seems an apt way of describing the analysis of research on social identity and the importance of belonging to groups presented in The Social Cure: Identity, Health and Wellbeing. This broad collection of literature, contributed to by over 30 leading researchers, shows that the metaphor does not adequately describe the extent of this body of research and the degree to which it is understated in everyday practice. A 'theory to practice gap' implies polar positions that require connecting, 'as if a third concept is needed which carries meaning from the theory side to the practice side, and vice versa' (Short, 2000, 